\title{
The mammalian INO80 complex is recruited to DNA damage sites in an ARP8
}

\section{dependent manner}

Shu-ichiro Kashiwaba ${ }^{1}$, Kazuyuki Kitahashi ${ }^{1}$, Takumi Watanabe ${ }^{1}$, Fumitoshi Onoda ${ }^{1}$, Masaya Ohtsu ${ }^{1}$, Yasufumi Murakami ${ }^{1}$

${ }^{1}$ Department of Biological Science and Technology, Faculty of Industrial Science and Technology, Tokyo University of Science, 2641 Yamazaki, Noda, Chiba 278-8510, Japan

Correspondence to;

Dr. Yasufumi Murakami

Biological Science and Technology

Faculty of Industrial Science and Technology

Tokyo University of Science

2641 Yamazaki, Noda-shi, Chiba 278-8510, JAPAN

Tel: 81-4-7124-1501 ex4408

FAX 81-4-7122-1360

Email: yasufumi@rs.noda.tus.ac.jp 


\section{Abstract}

Dynamic changes in chromatin structure are essential for efficient DNA processing such as transcription, replication, and DNA repair. Histone modifications and ATP-dependent chromatin remodeling are important for the alteration of chromatin structure. The INO80 chromatin remodeling complex plays an important role in HR-mediated repair of DNA double-strand breaks (DSBs). In yeast, the INO80 complex is recruited to the sites of DSBs via direct interaction with phosphorylated histone $\mathrm{H} 2 \mathrm{~A}$ and facilitates the processing of DSB ends. However, the function of the mammalian INO80 complex in DNA repair is mostly unknown. Here, we show that the mammalian INO80 complex is recruited to the laser-induced DNA damage sites in a phosphorylated $\mathrm{H} 2 \mathrm{AX}(\gamma \mathrm{H} 2 \mathrm{AX})$-independent manner. We also found that an actin-related protein, ARP8, is an important subunit that is required for the recruitment of the mammalian INO80 complex to the DNA damage sites, although the recruitment of the yeast INO80 complex requires its Nhp10 or Arp4 subunits. These results suggest that the mammalian INO80 complex is also recruited to DNA damage sites similarly to the yeast INO80 complex, but the mechanism of this recruitment may be different from that of the yeast INO80 complex. These findings provide new insights into the mechanisms of DNA repair in mammalian cells.

\section{Keywords}

chromatin remodeling, INO80, ARP8, DNA repair

\section{Introduction}

DNA double-strand breaks (DSBs) are the most serious type of DNA damage. Defects in DSB repair lead to genomic instability and tumorigenesis. DSBs are mainly repaired by either non-homologous end joining (NHEJ) or homologous 
recombination (HR). The accumulation of DNA repair proteins at DSBs is one of the important processes in both repair pathways [1]. Activation of the DNA damage checkpoint also requires the recruitment of several checkpoint proteins to DNA [2]. Genomic DNA is generally folded and packaged into the nucleus in eukaryotes, but highly condensed chromatin may be a barrier to the access of DNA repair proteins to the broken sites. Thus, chromatin structure must be changed at DNA damage sites. Histone modifications and ATP-dependent chromatin remodeling are the major mechanisms that alter chromatin structure and are known to be important for DNA repair and DNA damage responses [3].

In the early response to DSBs, histone $\mathrm{H} 2 \mathrm{AX}$ (H2A in yeast) is phosphorylated in the chromatin surrounding the DSBs by PI3K-related kinases like ATM/ATR. Phosphorylated H2AX $(\gamma \mathrm{H} 2 \mathrm{AX})$ forms nuclear foci in response to $\gamma$-irradiation, and participates in the accumulation and retention of numerous DNA repair factors at the DSBs [4]. ATP-dependent chromatin remodeling complexes use the energy of ATP hydrolysis to alter chromatin structure. Several ATP-dependent chromatin remodeling complexes of the SWI/SNF superfamily facilitate DNA repair in cooperation with modified histones such as $\gamma \mathrm{H} 2 \mathrm{AX}$.

The INO80 chromatin remodeling complex is known to be related to DNA repair in yeast, mammals, and plants [5,6,7]. The yeast INO80 complex is recruited to the DSBs via direct interaction of its Nhp10 or Arp4 subunits with phosphorylated $\mathrm{H} 2 \mathrm{~A}$, and participates in DNA end processing in HR repair $[8,9,10]$. On the other hand, mammalian INO80 facilitates HR-mediated DSB repair via expression of RAD51 and XRCC3 genes [11]. However, whether the mammalian INO80 complex also directly facilitates DSB repair is still unknown.

Recently, a comparison of the subunits between mammalian and yeast INO80 complexes revealed that these complexes share a set of subunits (INO80; two AAA+ ATPases, TIP49A and TIP49B; three actin-related proteins, ARP4, ARP5, and ARP8; and Ies2 and Ies6), and these subunits may participate in the conserved functions of INO80 complexes. On the other 
hand, yeast and mammalian INO80 complexes individually include several non-conserved subunits, and these subunits seemed to be related to functions that are unique to each species [12,13]. Interestingly, the Nhp10 subunit is required for the recruitment of the yeast INO80 complex to the DSBs, and the Ies4 subunit is related to the checkpoint activation in yeast, although these subunits are not conserved in the mammalian INO80 complex $[9,14]$. Thus, the behavior of the mammalian INO80 complex in DNA repair is thought to be different from that of the yeast INO80 complex. In this study, we demonstrate that the mammalian INO80 complex is also recruited to DNA damage sites, but the mechanism of this recruitment is different from that of the yeast INO80 complex.

\section{Materials and Methods}

\subsection{Cell culture}

HeLa and mouse embryonic fibroblasts (MEFs) were grown at $37{ }^{\circ} \mathrm{C}$ in DMEM (Nissui, Tokyo, Japan) supplemented with $10 \%$ fatal bovine serum, $2 \mathrm{mM} \mathrm{L-glutamine,} \mathrm{and} \mathrm{1 \%} \mathrm{penicillin} \mathrm{streptomycin} \mathrm{(Invitrogen,} \mathrm{Carlsbad,} \mathrm{CA)} \mathrm{under} \mathrm{a} \mathrm{5 \%} \mathrm{CO}_{2}$ environment. Immortalized MEFs were established by 3T3 protocol. MEFs which stably expressed FLAG-tagged mINO80 (MEF-mINO80) were established by retroviral infection.

FM3A cells were grown at $37{ }^{\circ} \mathrm{C}$ in RPMI 1640 (Invitrogen) supplemented with $10 \%$ calf serum under a $5 \% \mathrm{CO}_{2}$ environment.

\subsection{Antibodies}

A monoclonal antibody against mammalian INO80 was obtained from Biomatrix Research Inc. A polyclonal antibody 
against mammalian ARP8 was obtained from the serum of a rabbit that was immunized with recombinant mARP8. The full length of the cDNA coding for mARP8 was amplified and cloned into pET-32b(+) (Merck, Darmstadt, Germany), and recombinant mARP8 was expressed in E.coli strain BL21(DE3). Inclusion bodies containing mARP8 were washed and dissolved into denaturing buffer $\left(8 \mathrm{M}\right.$ urea, $100 \mathrm{mM} \mathrm{NaH}_{2} \mathrm{PO}_{4}, 10 \mathrm{mM}$ Tris-HCl, $\mathrm{pH}$ 8.0) followed by overnight dialysis against buffer containing $1 \mathrm{M}$ urea. The prepared antigen solution was mixed with Freund's complete adjuvant (Becton Dickinson, Franklin Lakes, NJ) and injected into a rabbit (Male, Japanese White, Japan SLC, Nagano, Japan). For the second to sixth immunizations, antigen solution was mixed with Freund's incomplete adjuvant (Becton Dickinson) and injected every two weeks. The antibodies were affinity purified from the rabbit serum. Rabbit anti-H2AX and mouse anti- $\gamma \mathrm{H} 2 \mathrm{AX}$ were purchased from Millipore. Rabbit anti- $\gamma \mathrm{H} 2 \mathrm{AX}$ (ab2893) was purchased from Abcam. HRP-conjugated goat anti-mouse IgG, HRP-conjugated goat anti-rabbit IgG, Alexa Fluor 488 goat anti-mouse IgG, and Alexa Fluor 594 anti-rabbit $\operatorname{IgG}$ were purchased from Invitrogen.

\subsection{Laser scissors treatment, immunofluorescent staining and western blotting}

Cells were seeded on 8-well chamber slides (Beckton Dickinson). The next day, the cells were treated with a $355 \mathrm{~nm}$ solid state UV laser using the MMI CellCut system. After laser treatment, the cells were allowed to recover for $5 \mathrm{~min}$ to $6 \mathrm{~h}$ at 37

${ }^{\circ} \mathrm{C}$. The cells were then fixed with $2 \%$ Paraformaldehyde (PFA) for $10 \mathrm{~min}$ at room temperature and permeabilized with 0.5\% Triton X-100 in phosphate-buffered saline (PBS) for 5 min on ice, followed by blocking with $10 \%$ goat serum in PBS for an hour at room temperature. After the blocking procedure, anti-INO80 and anti- $\gamma \mathrm{H} 2 \mathrm{AX}$ were mixed into $4 \%$ goat serum and applied to the cells, and the slides were incubated for an hour at room temperature followed by incubation with secondary antibodies (Alexa Fluor 488 goat anti-mouse IgG, Alexa Fluor 594 goat anti-rabbit IgG) in $4 \%$ goat serum 
containing $2 \mu \mathrm{g} / \mathrm{ml}$ DAPI (Dojindo, Kumamoto, Japan) for an hour at room temperature. Fluorescence images were generated with an Axiovert 200 fluorescence microscope (Carl Zeiss, Oberkochen, Germany).

Plasmid construction, retroviral infection, immunoblotting and quantitative RT-PCR (qRT-PCR)

These methods are described in the supplementary data.

\section{Results}

\subsection{The mammalian INO80 complex was recruited to the laser-induced DNA damage sites}

Previous studies showed that the mammalian INO80 complex is related to DNA repair [6,15]. However, it remains unclear whether mammalian INO80 is recruited to DNA damage sites and directly participate in DNA repair. To investigate whether the mammalian INO80 complex could be recruited to DNA damage sites like the yeast INO80 complex, we first produced a monoclonal antibody against endogenous mammalian INO80 protein. Using this monoclonal antibody, we detected human and mouse INO80 protein by immunoblotting (Fig. 1A) and immunostaining (Fig. 1B). Consistent with previous studies, mammalian INO80 was distributed broadly in the nucleus of undamaged cells. Then we introduced DNA damages into HeLa cells and mouse embryonic fibroblasts (MEFs) using laser scissors and performed immunostaining using anti-INO80 antibody. Phosphorylation of $\mathrm{H} 2 \mathrm{AX}(\gamma \mathrm{H} 2 \mathrm{AX})$ was observed along the paths of the laser 20 min after irradiation. Mammalian INO80 was co-localized with $\gamma \mathrm{H} 2 \mathrm{AX}$ in HeLa cells and MEFs, although most INO80 remained uniformly in the nucleus (Fig. 2A). To sharpen the signal of INO80 in MEF cells, we established MEFs that stably expressed FLAG-tagged mINO80 by retroviral infection (Supplementary Fig. 1). We named the cell line "MEF-mINO80." 
MEF-mINO80 cells were treated with laser scissors and immunostained with anti-INO80 and anti-FLAG M2 antibodies. In

MEF-mINO80 cells, we observed clearer staining of mINO80 at the DNA damage sites compared with normal MEFs (Figs.

1B, 2B). By using anti-FLAG M2 antibody, we confirmed that FLAG-tagged mINO80 was also recruited to the DNA damage sites (data not shown). These results suggest that the mammalian INO80 complex is recruited to DNA damage sites like the yeast INO80 complex.

\subsection{Phosphorylation of histone $\mathrm{H} 2 \mathrm{AX}$ was dispensable for the accumulation and retention of the mammalian INO80}

\section{complex at the laser-induced DNA damage sites}

In yeast, the INO80 complex is recruited to DSBs through the interaction of its Nhp10 or Arp4 subunits with phosphorylated $\mathrm{H} 2 \mathrm{~A}$ (corresponding to mammalian $\gamma \mathrm{H} 2 \mathrm{AX}$ ) [8,9]. To investigate the dependency of the phosphorylation of $\mathrm{H} 2 \mathrm{AX}$ on the recruitment of mammalian INO80 to DNA damage sites, we examined INO80 staining after laser scissors treatment in $\mathrm{H}_{2} \mathrm{AX}^{-{ }^{-}}$MEFs (Supplementary Fig. 2). Similar to HeLa cells, mINO80 was observed at the sites of the laser-induced DNA damages in $H 2 A X^{-/-}$MEFs at 20 min after irradiation (Fig. 3A).

Although several DNA repair proteins including 53BP1, BRCA1, and NBS1 migrate to the DSBs in a $\gamma \mathrm{H} 2 \mathrm{AX}$-independent manner, $\gamma \mathrm{H} 2 \mathrm{AX}$ are required for the retention of these proteins at the DSBs [16]. Thus, we investigated whether $\gamma \mathrm{H} 2 \mathrm{AX}$ is required for the retention of mammalian INO80 at DNA damage sites. $H 2 A X^{+/+}$and $H 2 A X^{-/-}$MEFs were treated with laser scissors and allowed to recover for $5 \mathrm{~min}$ to $6 \mathrm{~h}$. Mammalian INO80 was localized at the DNA damage sites 5 min after laser scissors treatment and was retained for at least $6 \mathrm{~h}$ in both $H 2 A X^{+/+}$and $H 2 A X^{-/}$MEFs (Figs. 3B, C). Taken together, these results suggest that phosphorylation of histone $\mathrm{H} 2 \mathrm{AX}$ is dispensable for the recruitment and retention of mammalian INO80 complex to the laser-induced DNA damage sites. 


\subsection{ARP8 is required for the accumulation of the mammalian INO80 complex to the laser-induced DNA damage}

sites

Since the manner of recruitment of the mammalian INO80 complex to DNA damage sites might be different from that of the yeast INO80 complex, we investigated which subunit(s) of the mammalian INO80 complex is (are) required for the recruitment. To identify the subunit(s), we performed an RNAi screening using stable knocked-down cell lines of the genes coding for the subunits of the mammalian INO80 complex. The mammalian INO80 complex consists of at least 15 proteins. INO80, RUVBL1, RUVBL2, ARP4 (BAF53a), ARP5, ARP8, IES2 and IES6 are conserved between the yeast and mammalian INO80 complexes. INO80D, INO80E, MCRS1, NFRKB, TFPT, UCH37, and YY1 are included only in the mammalian INO80 complex, and may be related to mammalian-specific functions [12]. On the other hand, Nhp10 is not conserved in the mammalian INO80 complex; thus, we speculated that some of the non-conserved subunits may be required for the recruitment of mammalian INO80 to DNA damage sites. First, we produced seven MEF-mINO80 cell lines in which each gene coding for the non-conserved subunits was respectively knocked-down by retroviral infection. The knock-down efficiencies of the target gene in each cell line were monitored by qRT-PCR (Supplementary Fig. 3). These cell lines were treated with laser scissors and immunostained with anti-INO80. However, knock-downs of these genes including Yyl, which is known to be related to DSB repair [6], did not substantially affect the migration of mINO80 (Fig. 4A, C). We next produced seven cell lines in which the genes coding for the conserved subunits other than mINO80 were stably knocked-down. The knock-down efficiencies were monitored by qRT-PCR and immunoblotting (Supplementary Fig. 3). In yeast, Arp4 is found in the INO80, SWR1, and NuA4 chromatin remodeling complexes [8,17], and participates in the recruitment of these complexes to DSBs. However, Arp4 knocked-down cells did not show a significant reduction in the 
recruitment of mINO80 to the sites of DNA damage in this study. Interestingly, the accumulation of mINO80 to the DNA damage sites was reduced in the cells in which the gene coding for ARP8, which is an actin-related-protein like ARP4, was knocked-down (Figs. 4B, C). On the other hand, significant reduction of the recruitment of mINO80 was not observed in the cell lines that knocked-down the genes coding other subunits, including another actin-related protein, ARP5 (Figs. 4B, C). These results suggest that ARP8 is required for the recruitment of the mammalian INO80 complex to the laser-induced DNA damage sites.

\section{Discussion}

Previous studies revealed that the mammalian INO80 chromatin remodeling complex is related to DNA repair $[6,11,15]$, but the details of its mechanism are mostly unknown. Several DNA repair proteins form complexes and facilitate the recognition of DNA lesions or DNA repair processes $[18,19]$. Thus, it is important to identify the subunit(s) that is (are) required for the recruitment of the mammalian INO80 complex to DNA damage sites. In this study, we prepared a specific monoclonal antibody against mammalian INO80, and investigated whether mammalian INO80 accumulates at damaged DNA. Many DNA repair proteins accumulate at the IR-induced foci and co-localize with $\gamma \mathrm{H} 2 \mathrm{AX}[1,4]$. Hence, we investigated whether mammalian INO80 also co-localize with $\gamma \mathrm{H} 2 \mathrm{AX}$ at IR-induced foci. However, like undamaged cells, mammalian INO80 was distributed broadly in the nucleus (data not shown). The same result was previously reported [15]. Next, we introduce DNA damages using laser scissors, and we then detected mammalian INO80 at the laser-induced DNA damage sites. Laser scissors could easily generate DNA damages along the path of the laser, and have been used in several previous studies. Several proteins such as INO80 and Ku proteins are recruited to damaged DNA, although these proteins do not form distinct damage-induced foci. Thus, laser scissors also enable us to detect the recruitment of such proteins at 
damaged DNA [20,21].

A previous study indicated that loss of mammalian INO80 causes defects in HR-mediated DSB repair [6]. Our results, combined with this previous result, suggest that the mammalian INO80 complex may be recruited to the DSBs and directly participate in DSB repair. HR repair mainly occurs in the late S/G2 phase and several HR-related proteins such as RAD51 and BRCA1 accumulate at the DSBs only in this phase [22,23]. However, we detected mammalian INO80 at the DNA damage sites in all interphase cells; therefore, mammalian INO80 may be recruited to DNA damage sites also in phases other than the late S/G2 phase. In addition, laser scissors may mainly generate DSBs, but they may also generate other types of DNA damage. Thus, our result raises the possibility that the mammalian INO80 complex may also be related to NHEJ or other types of DNA repair. Indeed, the yeast INO80 complex is also known to be related to NHEJ.

In yeast, the INO80 complex is recruited to the DSBs through the direct interaction of its Nhp10 or Arp4 subunits with phosphorylated histone $\mathrm{H} 2 \mathrm{~A}[8,9]$. However, the ortholog of yeast Nhp10 does not exist in mammals and we believe that the manner of recruitment of the mammalian INO80 complex is different from that of the yeast INO80 complex. In this study, we have presented two novel findings related to the recruitment of the mammalian INO80 complex to DNA damage sites. First, mammalian INO80 is recruited to the laser-induced DNA damage sites in a $\gamma \mathrm{H} 2 \mathrm{AX}$-independent manner. Thus, the recruitment of the mammalian INO80 complex may require other chromatin proteins. Second, the ARP8 subunit participates in the recruitment of mammalian INO80. Interestingly, a yeast mutant with a defect in Arp8 does not exhibits significant reductions in either the recruitment of the INO80 complex to DSBs or the interaction with phosphorylated H2A [9,10]. Furthermore, the amino acid sequence identity between mammalian ARP8 and yeast Arp8 is only about 20\%, although mammalian ARP8 was identified as the ortholog of yeast Arp8. Thus, it is likely that mammalian ARP8 acquired a new ability during evolution, thereby facilitating the recruitment of the INO80 complex to damaged DNA. Yeast Arp8 has 
an ability to interact with core histones [24], and mammalian histone H2B Ser14 is also phosphorylated in DNA damage responses [25]. Therefore, it is possible that the mammalian INO80 complex may be recruited to damaged DNA via interaction not with $\gamma \mathrm{H} 2 \mathrm{AX}$ but with other modified core histones such as phosphorylated $\mathrm{H} 2 \mathrm{~B}$. On the other hand, a recent study showed that mammalian INO80 interacts with $\gamma \mathrm{H} 2 \mathrm{AX}$ and facilitates the damage-induced phosphorylation of $\mathrm{H} 2 \mathrm{AX}$ [15]. Taken together, these results suggest that although the mammalian INO80 complex is recruited to damaged DNA in a $\gamma \mathrm{H} 2 \mathrm{AX}$-independent manner, this complex may interact with $\gamma \mathrm{H} 2 \mathrm{AX}$ at damaged DNA, and cooperate with $\gamma \mathrm{H} 2 \mathrm{AX}$ to facilitate DNA repair. In a finding that supports this possibility, NBS1 was found to be recruited to the DSBs independently of $\mathrm{H} 2 \mathrm{AX}$, but to directly interact with $\gamma \mathrm{H} 2 \mathrm{AX}$ at the DSBs [26].

Actin-related proteins are included in many chromatin remodeling complexes. The INO80 complex includes three actin-related proteins, namely ARP4, ARP5 and ARP8. The sequences of actin-related proteins are highly diverse except for several domains similar to actin, and are thought to each have specific roles. ARP4 is also included in the SRCAP and TRRAP (homologous to yeast SWR and NuA4, respectively) complexes, and is thought to participate in the functions common to these complexes. In contrast, ARP5 and ARP8 are included only in the INO80 complex and may be related to the functions that are specific to the INO80 complex [12,13]. In humans, both ARP5 and ARP8 localize to the nuclei of interphase cells. However, during mitosis, although ARP5 is excluded from chromatin, ARP8 accumulates on the chromatin [27]. On the other hand, both Arp5 and Arp8 are known to be related to DNA repair in yeast [10,24], but functional differences between Arp5 and Arp8 in DNA repair are still unclear. In this study, the recruitment of mammalian INO80 to the laser-induced DNA damage sites is markedly reduced only in Arp 8 knocked-down cells. This result raises the possibility that ARP8 and ARP5 function differently in DNA repair. A recent study showed that hARP5 knocked-down cells exhibit hypersensitivity to DNA damage [15]. Interestingly, like the yeast strain deleted for Nhp10, Arp8 knocked-down cells did 
not exhibit serious sensitivity to DNA damage (data not shown), although the recruitment of the INO80 complex to laser-induced DNA damage sites was reduced significantly. It is conceivable that ARP8 is required for the recruitment, and ARP5 is required for the DNA repair functions of the INO80 complex.

It is noteworthy that the recruitment of INO80 to the DNA damage sites did not completely disappear in Arp8 knocked-down cells. Therefore, our results suggest that ARP8 is the major subunit that participates in the recruitment of the mammalian INO80 complex to damaged DNA, whereas we could not exclude the possibility that ARP8 controls the recruitment in cooperation with other subunits.

Although the most recent study revealed that mammalian INO80 indirectly facilitates DSB repair via the expression of RAD54B and XRCC3 genes [11], the molecular function of the mammalian INO80 complex at DNA damage sites is still unclear. We are now investigating the molecular functions of the mammalian INO80 complex in DNA repair by using I-sceI endonuclease which is able to generate a single DSB at the particular site of genomic DNA. The relationship of chromatin remodeling with both genome maintenance and diseases that are related to DNA damage is an important research topic. Indeed, recent studies reported that the mammalian SWI/SNF chromatin remodeling complexes (which is known to facilitate DSB repair like the INO80 complex) is related to tumorigenesis [28]. We believe that further analyses will reveal the relationship between the mammalian INO80 complex and diseases related to DNA damage in the near future.

\section{Acknowledgement}

We thank Dr. Toshio Kitamura for Plat-E packaging cells, and Dr. Andre Nussensweig for $H 2 A X^{-/}$MEFs. This work has been supported by the grants supplied by the Ministry of Education, Culture, Sports, Science and Technology, the Japan 
Science and Technology Agency, and Bio Matrix Research Inc.

\section{References}

[1] J.W. Harper, S.J. Elledge, The DNA damage response: Ten years after, Mol. Cell 28 (2007) 739-745.

[2] E. Soutoglou, T. Misteli, Activation of the cellular DNA damage response in the absence of DNA lesions, Science 320 (2008) $1507-1510$.

[3] M. Downey, D. Durocher, Chromatin and DNA repair: the benefits of relaxation, Nat. Cell Biol. 8 (2006) 9-10.

[4] T.T. Paull, E.P. Rogakou, V. Yamazaki et al., A critical role for histone H2AX in recruitment of repair factors to nuclear foci after DNA damage, Curr. Biol. 10 (2000) 886-895.

[5] X.T. Shen, G. Mizuguchi, A. Hamiche, C. Wu, A chromatin remodelling complex involved in transcription and DNA processing, Nature 406 (2000) 541-544.

[6] S. Wu, Y.J. Shi, P. Mulligan et al., A YY1-INO80 complex regulates genomic stability through homologous recombination-based repair, Nat. Struct. Mol. Biol. 14 (2007) 1165-1172.

[7] O. Fritsch, G. Benvenuto, C. Bowler et al., The INO80 protein controls homologous recombination in Arabidopsis thaliana, Mol. Cell 16 (2004) 479-485.

[8] J.A. Downs, S. Allard, O. Jobin-Robitaille et al., Binding of chromatin-modifying activities to phosphorylated histone H2A at DNA damage sites, Mol. Cell 16 (2004) 979-990.

[9] A.J. Morrison, J. Highland, N.J. Krogan et al., INO80 and gamma-H2AX interaction links ATP-dependent chromatin remodeling to DNA damage repair, Cell 119 (2004) 767-775. 
[10] H. van Attikum, O. Fritsch, B. Hohn, S.M. Gasser, Recruitment of the INO80 complex by H2A phosphorylation links ATP-dependent chromatin remodeling with DNA double-strand break repair, Cell 119 (2004) 777-788.

[11] E. Park, S. Hur, J. Kwon, Human INO80 chromatin remodeling complex contributes to DNA double strand break repair via the expression of Rad54B and XRCC3 genes, Biochem. J. (in press).

[12] R.C. Conaway, J.W. Conaway, The INO80 chromatin remodeling complex in transcription, replication and repair, Trends Biochem. Sci. 34 (2009) 71-77.

[13] J.Y. Jin, Y. Cai, T. Yao et al., A mammalian chromatin remodeling complex with similarities to the yeast INO80 complex, J. Biol. Chem. 280 (2005) 41207-41212.

[14] A.J. Morrison, J.A. Kim, M.D. Person et al., Mec1/Tel1 phosphorylation of the INO80 Chromatin Remodeling Complex Influences DNA damage checkpoint responses, Cell 130 (2007) 499-511.

[15] K. Kitayama, M. Kamo, Y. Oma et al., The human actin-related protein hArp5: Nucleo-cytoplasmic shuttling and involvement in DNA repair, Exp. Cell Res. 315 (2009) 206-217.

[16] A. Celeste, O. Fernandez-Capetillo, M.J. Kruhlak et al., Histone H2AX phosphorylation is dispensable for the initial recognition of DNA breaks, Nat. Cell Biol. 5 (2003) 675-U651.

[17] H. Ogiwara, A. Ui, S. Kawashima et al., Actin-related protein Arp4 functions in kinetochore assembly, Nucleic Acids Res. 35 (2007) 3109-3117.

[18] J.P. Carney, R.S. Maser, H. Olivares et al., The hMre11/hRad50 protein complex and Nijmegen breakage syndrome: Linkage of double-strand break repair to the cellular DNA damage response, Cell 93 (1998) 477-486.

[19] B.B.S. Zhou, S.J. Elledge, The DNA damage response: putting checkpoints in perspective, Nature 408 (2000) 433-439.

[20] Y. Enokido, T. Tamura, H. Ito et al., Mutant huntingtin impairs Ku70-mediated DNA repair, J. Cell Biol. 189 (2010) 
425-443.

[21] J.S. Kim, T.B. Krasieva, H. Kurumizaka et al., Independent and sequential recruitment of NHEJ and HR factors to DNA damage sites in mammalian cells, J. Cell Biol. 170 (2005) 341-347.

[22] R. Scully, J.J. Chen, R.L. Ochs, K et al., Feunteun, D.M. Livingston, Dynamic changes of BRCA1 subnuclear location and phosphorylation state are initiated by DNA damage, Cell 90 (1997) 425-435.

[23] S.S.F. Yuan, H.L. Chang, E. Lee, Ionizing radiation-induced Rad51 nuclear focus formation is cell cycle-regulated and defective in both ATM(-/-) and c-Abl(-/-) cells, Mutat. Res. Fundam. Mol. Mech. Mutag. 525 (2003) 85-92.

[24] X.T. Shen, R. Ranallo, E. Choi, C. Wu, Involvement of actin-related proteins in ATP-dependent chromatin remodeling, Mol. Cell 12 (2003) 147-155.

[25] O. Fernandez-Capetillo, C.D. Allis, A. Nussenzweig, Phosphorylation of histone H2B at DNA double-strand breaks, J. Exp. Med. 199 (2004) 1671-1677.

[26] J. Kobayashi, H. Tauchi, S. Sakamoto et al., NBS1 localizes to gamma-H2AX foci through interaction with the FHA/BRCT domain, Curr. Biol. 12 (2002) 1846-1851.

[27] N. Aoyama, A. Oka, K. Kitayama et al., The actin-related protein hArp8 accumulates on the mitotic chromosomes and functions in chromosome alignment, Exp. Cell Res. 314 (2008) 859-868.

[28] A.K.C. Wong, F. Shanahan, Y. Chen et al., BRG1, a component of the SWI-SNF complex, is mutated in multiple human tumor cell lines, Cancer Res. 60 (2000) 6171-6177. 


\section{Figure legends}

\section{Figure 1 Evaluation of anti-INO80 monoclonal antibody.}

The specificity of the anti-INO80 monoclonal antibody was evaluated by immunoblotting and immunostaining. (A) The whole cell lysates from HeLa and FM3A cells were separated by SDS-PAGE, and immunoblotted with anti-INO80 antibody (left). HeLa cells were transfected with pcDNA3-FLAG-hINO80 or pcDNA3, and the whole cell lysates from these cells were immunoblotted with anti-FLAG M2 antibody (right). (B) mINO80 knocked-down FM3A cells were established by retroviral infection. The whole cell lysate from this strain was separated by SDS-PAGE, and immunoblotting with anti-INO80. FM3A cells which express shRNA for the GFP gene were used as a control. (C) Intercellular localization of mammalian INO80. HeLa cells, MEFs and FLAG-mINO80-expressing MEFs (MEF-mINO80) were fixed with 2\% PFA for 10 min. Cells were then stained with anti-INO80 antibody (green) and DAPI (blue). Scale bar, $10 \mu \mathrm{m}$.

Figure 2 Mammalian INO80 accumulates at the laser-induced DNA damage sites.

HeLa cells, MEFs and MEF-mINO80 cells were treated with laser scissors. The cells were then cultured for 20 min, and fixed with 2\% PFA for 10 min. After permeabilization and a blocking procedure, the cells were stained with anti-INO80 (green), anti- $\gamma \mathrm{H} 2 \mathrm{AX}$ (red), and DAPI (blue). Scale bar, $10 \mu \mathrm{m}$.

Figure 3 H2AX does not affect the accumulation and the retention of mammalian INO80 at the laser-induced damage sites.

(A) INO80 staining at the laser-induced DNA damage sites in $H 2 A X^{-/}$MEFs. $H 2 A X^{+/+}$MEFs were treated with laser scissors along the defined paths. After $20 \mathrm{~min}$, the cells were stained with anti-INO80 antibody (green) and DAPI (blue). 
(B) $H 2 A X^{+/+}$and $H 2 A X^{-/-}$MEFs were damaged by laser scissors treatment. After $5 \min$ or $6 \mathrm{~h}$, the cells were fixed and stained with anti-INO80 antibody (green). (C) The recruitment intensity of INO80 was quantified with IMAGE J software. In both cell lines, 20 laser-treated cells were randomly chosen, and the mean value of INO80 staining at the DNA damage sites was quantified at each defined time point. The quantified value was then normalized to the mean value of the undamaged area, and the normalized value was defined as recruitment intensity.

\section{Figure 4 RNAi screening of crucial subunits for the recruitment of mammalian INO80.}

(A) INO80 staining at the laser-induced DNA damage sites in the cells that expressed shRNA for each gene that encodes the non-conserved (left panel) or conserved (right panel) subunits of the mammalian INO80 complex. These cells were treated with laser scissors; and then immunostained with anti-INO80 (green) and anti- $\gamma \mathrm{H} 2 \mathrm{AX}$ (red) antibodies. (B) In each cell lines, at least 20 laser-treated cells were randomly chosen, and the recruitment intensity of INO80 was quantified with

IMAGE J software. 
Fig. 1

A

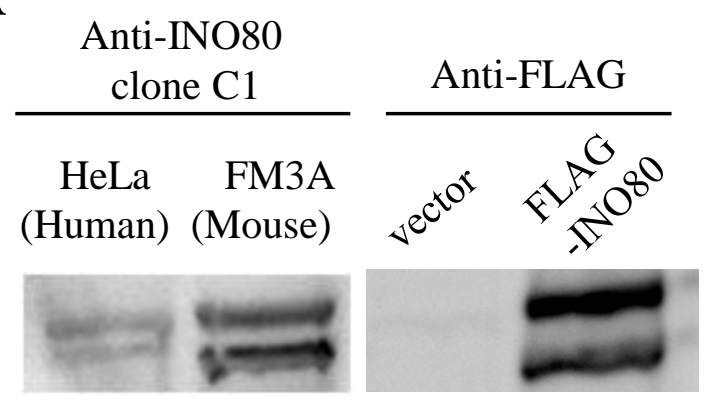

B

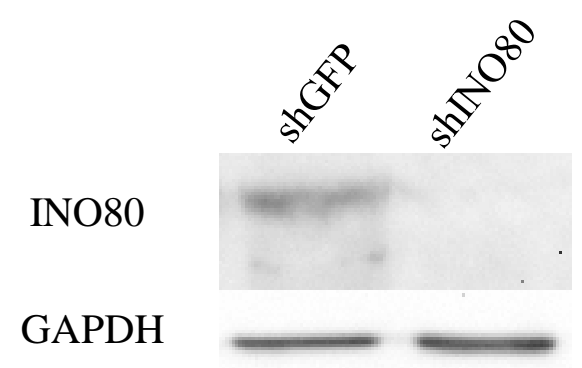

GAPDH

C

DAPI

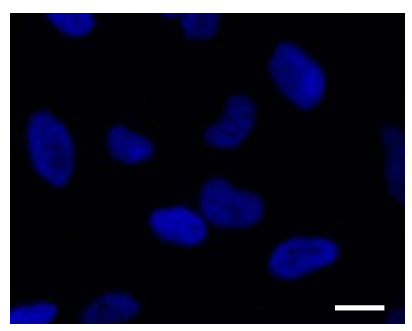

INO80
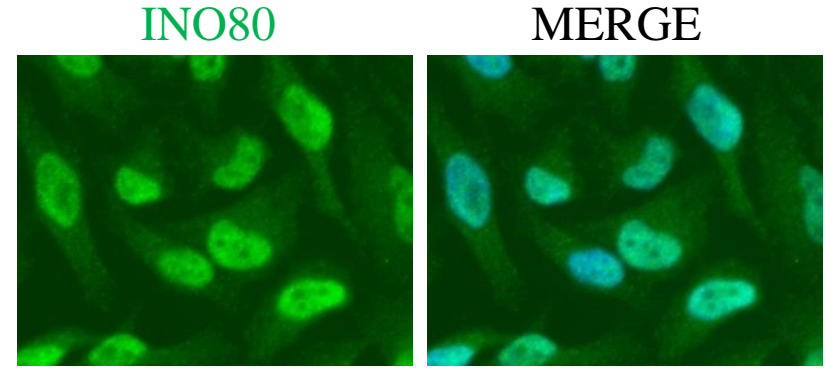

MEFs
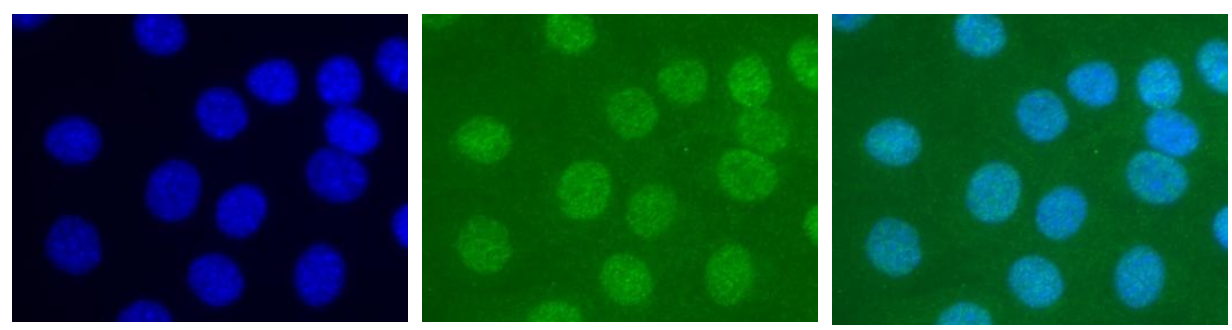

MEF -mINO80
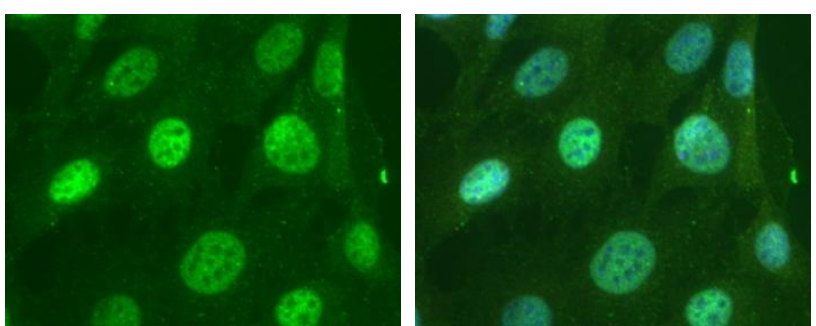
Fig. 2

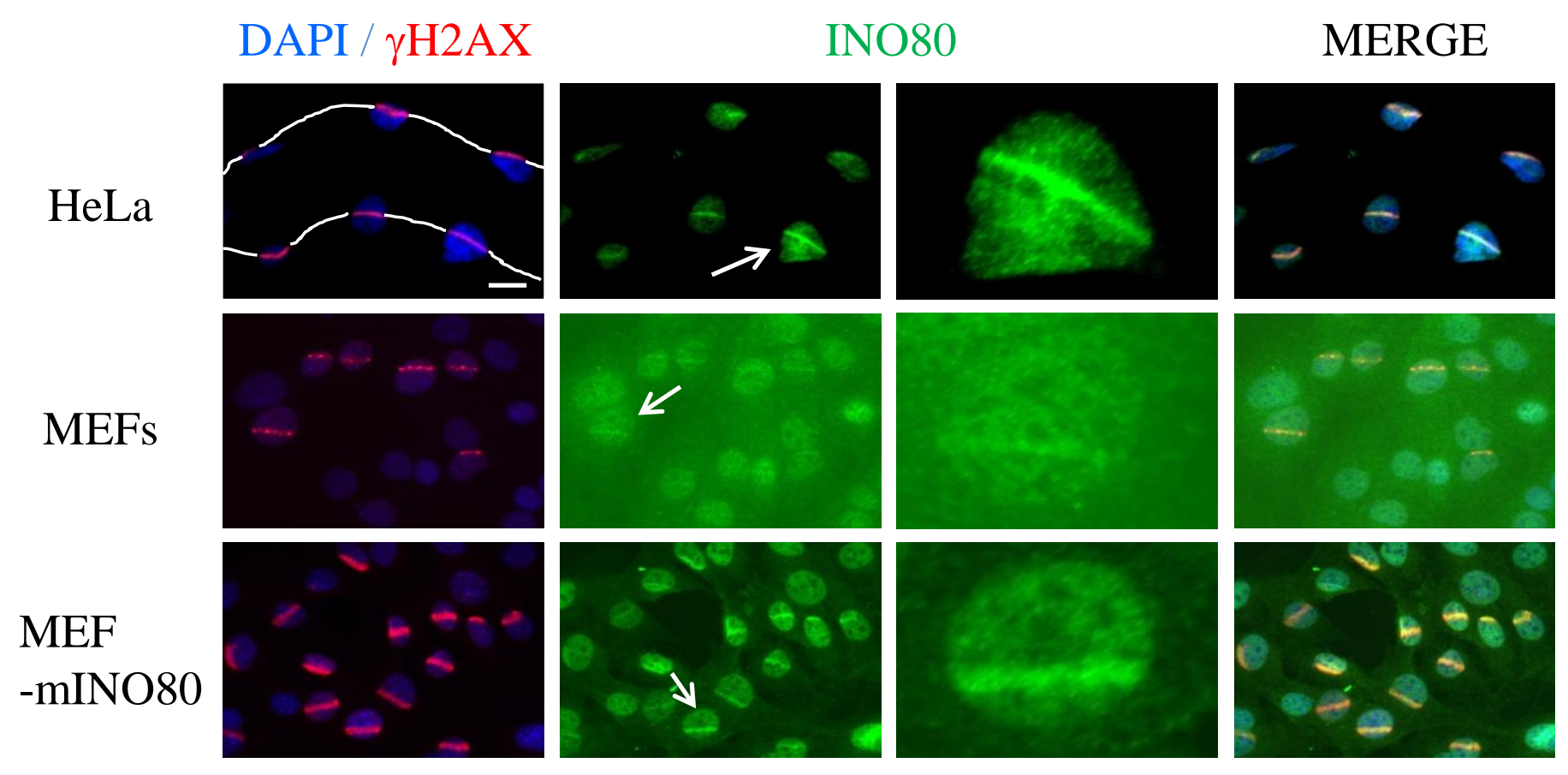


Fig. 3

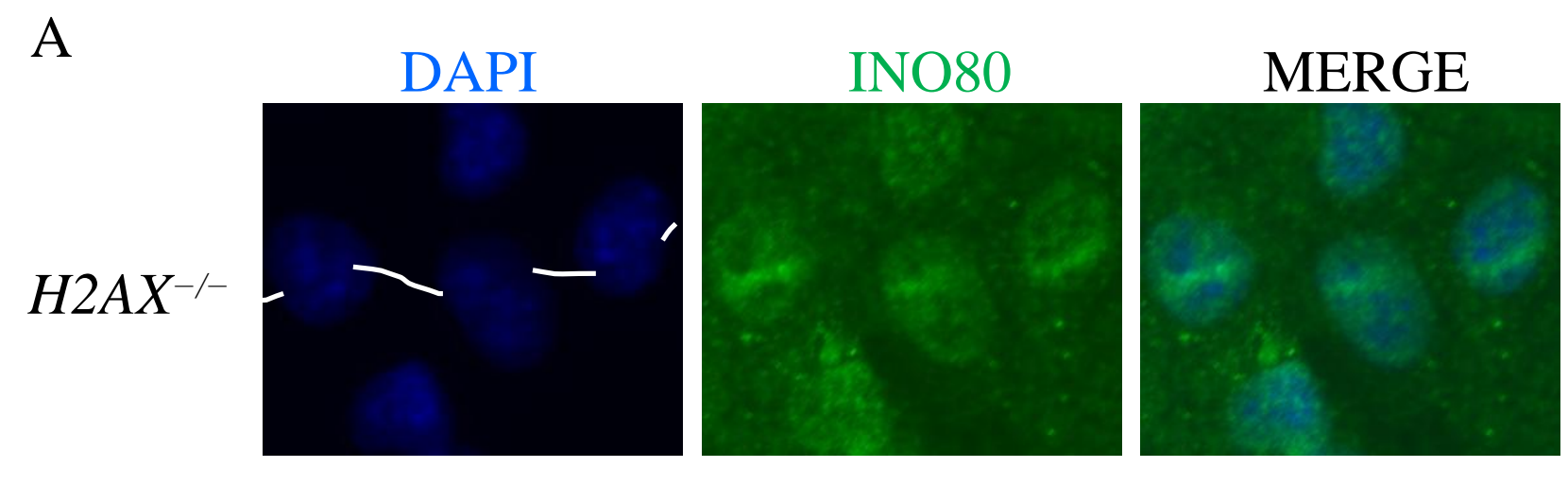

B

$H 2 A X^{+/+}$

5 min

$6 \mathrm{~h}$
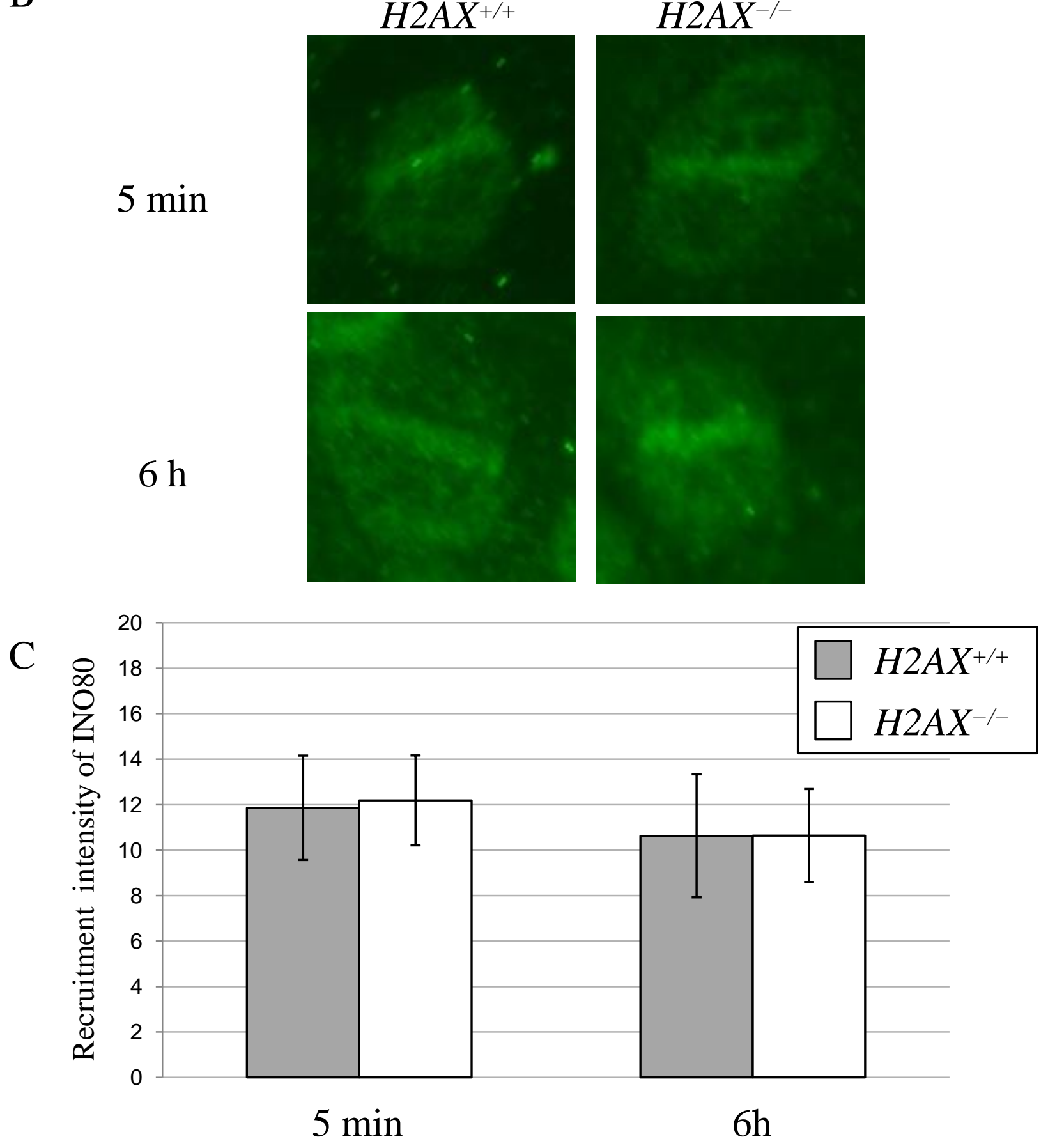

$H 2 A X^{-/-}$
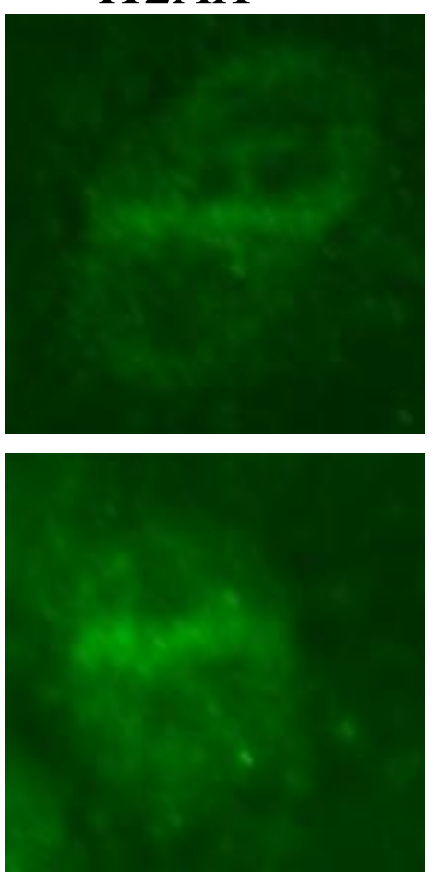

h 
Fig. 4

A

DAPI / $\gamma \mathrm{H} 2 \mathrm{AX}$

shGL2

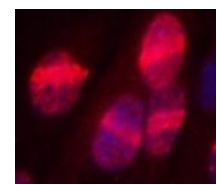

shYy1
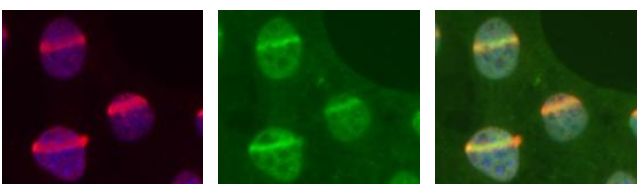

shUchl5

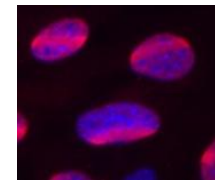

shMcrs1
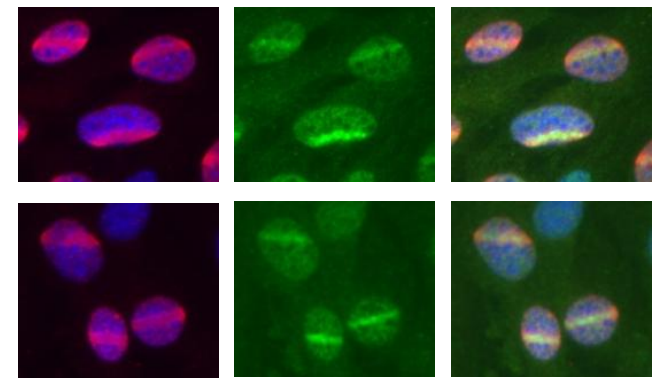

B

shArp4

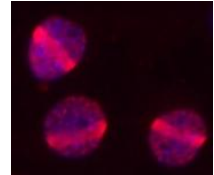

shArp5

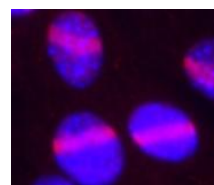

shArp8-1

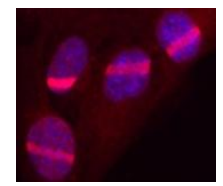

shArp8-2

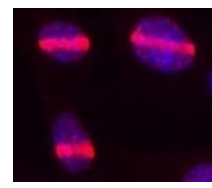

INO80
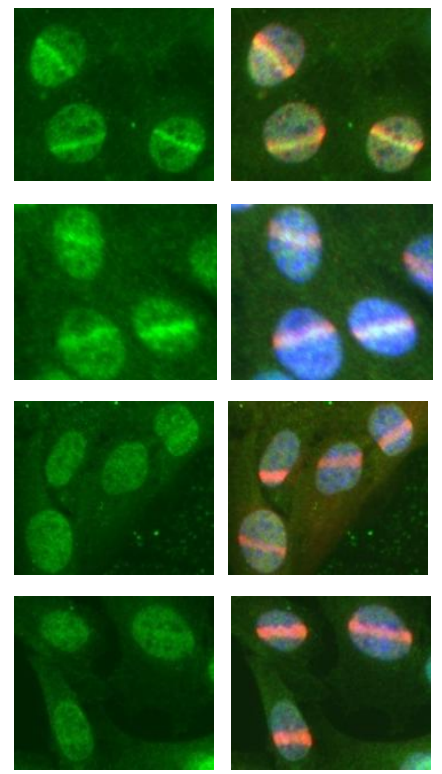

MERGE
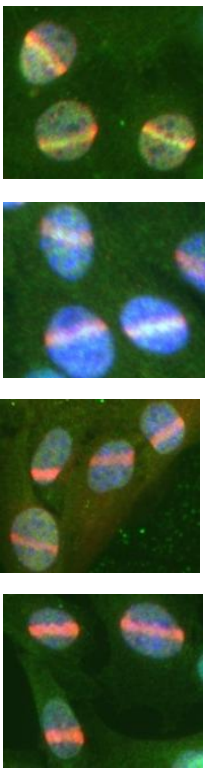

C

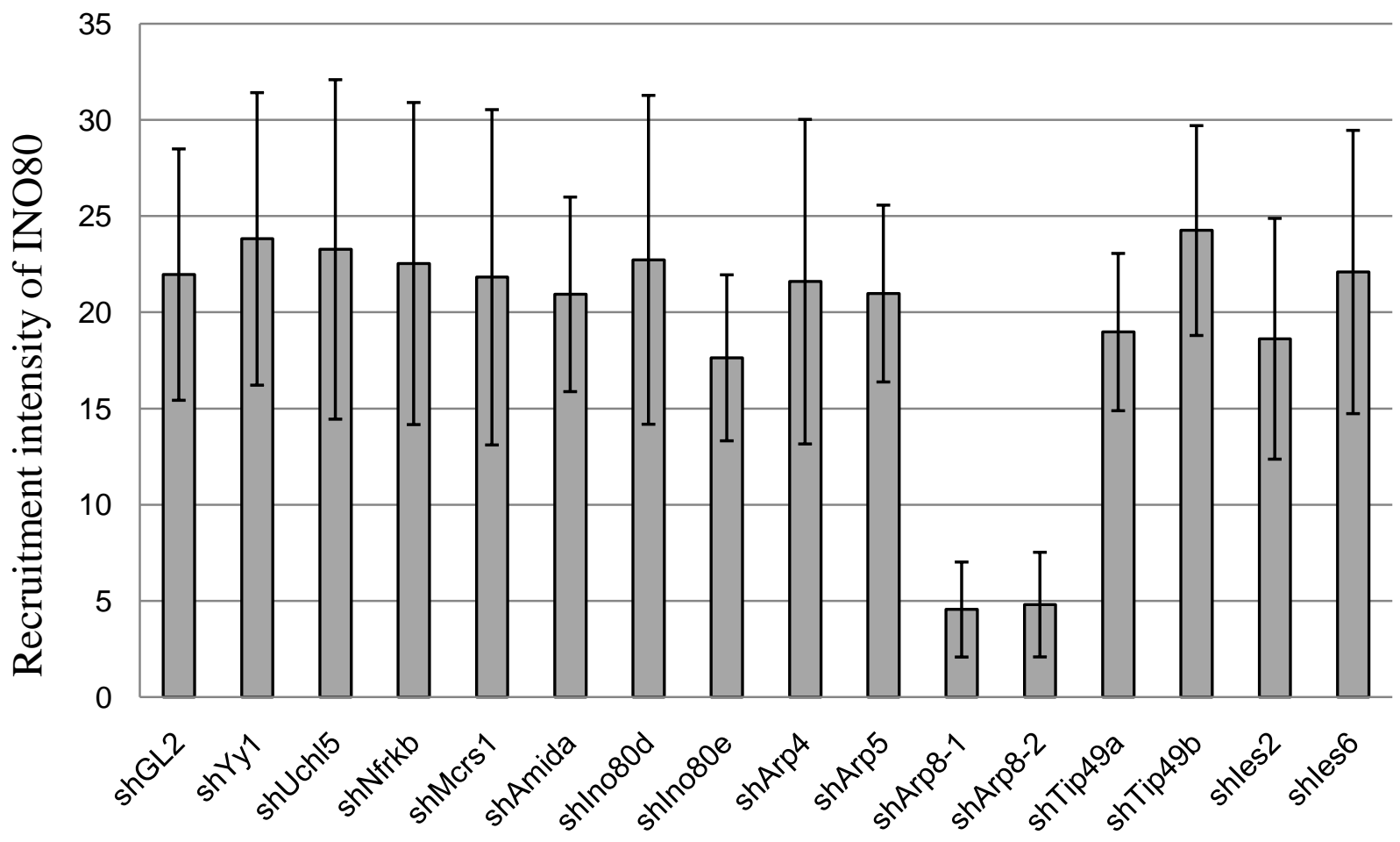

